\title{
Management of Pod Borer (Helicoverpa armigera Hubner) Infesting Marigold (Tagetes erecta)
}

\author{
Suheel Ahmad Ganai", Hafeez Ahmad, Sonika Sharma, Nadeya Khaliq, \\ Thanlass Norboo, Divya Chaand and Shallu Raina
}

\author{
Block No. 6, Division of Entomology, FoA Main Campus, SKUAST-J, Chatha, \\ Jammu -180 009, J\&K, India \\ *Corresponding author
}

\section{A B S T R A C T}

\begin{tabular}{|l|}
\hline Ke y w or d s \\
Tagetes erecta, \\
Helicoverpa \\
armigera, \\
Management, \\
Efficacy, \\
Carbosulfan. \\
\hline Article Info \\
\hline Accepted: \\
04 July 2017 \\
Available Online: \\
10 September 2017 \\
\hline
\end{tabular}

\section{Introduction}

Floriculture being an important agri-business sector contributes widely to the Indian economy through opportunities in terms of employment, income generation earning foreign exchange and empowerment, thus raising the socio - economic status in both rural and urban areas (Pandey et al., 2010). Owing to steady increase in demand of flower, floriculture has become one of the important Commercial trades in Agriculture. Hence commercial floriculture has emerged as hi-tech activity-taking place under controlled climatic conditions inside greenhouse. Commercial floriculture is becoming important from the export angle. Indian floriculture industry has been shifting from traditional flowers to cut flowers for export purposes. The liberalized economy has given an impetus to the Indian entrepreneurs for establishing export oriented floriculture units under controlled climatic conditions. In India, flowers occupied an area of 232.74 thousand hectares with a production of 1.729 million tonnes loose flowers and 76.73 million tonnes cut flowers during 2012-13 (Anonymous, 2016) and marigold ranks first among the loose flowers followed by chrysanthemum, jasmine, tuberose, 
crossandra and barleria (Bhattacharjee, 2001). Area under marigold cultivation is increasing steadily due to its heavy demand in domestic as well in international market. In India, marigold is one of the most commonly grown cut flower and extensively used in religious and social occasions in the one form or other. Large scale intensive cultivation of marigold has destabilized the crop-pest equilibrium and invited a number of problems. Attack by insects, mites and other pests are one of the important bottlenecks for successful production of these crops.

The attack by insects, mites and other pests increased manifold in the recent past. Various species of insect-pests viz. thrips, aphids, leaf hoppers, scale insects, mealy bugs, leaf miners, caterpillars, cut worms and chaffer beetles attack marigold (Anonymous, 2014).

Moreover, some new pests are appearing to invade the crop mainly due to the recent climate change and shift in crop culture methods. Several workers have reported a number of pests infesting the crop from various parts of the country. Information on pests infesting a crop is an essential prerequisite for developing a suitable pest management strategy particularly in the context of ever changing pest scenario. Keeping in view its economic importance this study was conducted.

\section{Materials and Methods}

For the pod borer management, a trial was laid out in the randomized block design with three replications and recommended agronomic practices for marigold plants were followed. Variety "Pusa Narangi Gainda" was used and ten insecticidal formulations including control were tested. Observations on the pod borer population from the selected plants were recorded before and after 1, 7 and 14 days of spray using a knapsack sprayer. In control plots only water was sprayed. The sprayer was rinsed carefully after each spray. Data thus obtained were analysed statistically and the efficacy was worked out.

\section{Results and Discussion}

Efficacy of the insecticides for the management of the pest revealed that all the insecticidal treatments at 1,7 and 14 days after spray were superior to control in reducing the pod borer population (Tables 1 and 2) during 2014 and 2015. The observations recorded on $14^{\text {th }}$ day after spray revealed that all the treatments proved significantly superior over control. Carbosulfan (0.67 and 0.33 pod borer larvae plant $^{-1}$ ) and novaluron (1.05 and 0.62 pod borer larvae plant $^{-1}$ ) were found best treatment in reducing the mite population which were statistically at par with each other. The treatments viz. bifenthrin (2.00 and 1.67 pod borer larvae plant $^{-1}$ ) and methyl-odemeton (3.38 and 2.28 pod borer larvae plant $^{-1}$ ) were statistically at par with each other.

Imidacloprid (4.33 and 3.56 pod borer larvae plant $\left.^{-1}\right)$, chlorpyriphos dust (5.63 and 4.48 pod borer larvae plant $\left.{ }^{-1}\right)$, thiamethoxam (7.05 and 6.07 pod borer larvae plant $^{-1}$ ), propargite (7.35 and 6.20 pod borer larvae plant ${ }^{-1}$ ) and neem oil ( 8.30 and 7.24 pod borer larvae plant $^{-1}$ ) were found to be least effective against the pod borer.

The pooled data during 2014 and 2015 (Table 3 , First spray) revealed that there was no significant difference between the treatments one day before spray. The observations recorded on $1^{\text {st }}$ day after spray revealed that all the treatments proved significantly superior over control. Carbosulfan (4.01 pod borer larvae plant ${ }^{-1}$ ) was found to be most effective treatment in reducing the pod borer population which was statistically at par with 
novaluron (5.46 pod borer larvae plant ${ }^{-1}$ ). The treatments viz. bifenthrin (5.66 pod borer larvae plant $\left.{ }^{-1}\right)$, methyl-o-demeton (8.13 pod borer larvae plant $^{-1}$ ), imidacloprid (8.38 pod borer larvae plant $^{-1}$ ), chlorpyriphos dust (10.15 pod borer larvae plant ${ }^{-1}$ ) and thiamethoxam (10.67 pod borer larvae plant ${ }^{-1}$ ) were statistically at par with each other. Propargite (11.48 pod borer larvae plant $^{-1}$ ) and neem oil (11.62 pod borer larvae plant ${ }^{-1}$ ) were found to be least effective against the pod borer. The observations recorded on $7^{\text {th }}$ day after spray revealed that all the treatments proved significantly superior over control. Carbosulfan (1.61 pod borer larvae plant ${ }^{-1}$ ) was found to be most effective treatment in reducing the pod borer population which was statistically at par with novaluron (2.47 pod borer larvae plant $\left.^{-1}\right)$. The treatments viz. bifenthrin (3.49 pod borer larvae plant $^{-1}$ ), methyl-o-demeton (4.91 pod borer larvae plant $^{-1}$ ), imidacloprid (6.13 pod borer larvae plant $^{-1}$ ), chlorpyriphos dust (7.52 pod borer larvae plant ${ }^{-1}$ ) and thiamethoxam (8.46 pod borer larvae plant $^{-1}$ ) were statistically at par with each other. Propargite (9.46 pod borer larvae plant ${ }^{-1}$ ) and neem oil (10.50 pod borer larvae plant ${ }^{-1}$ ) were found to be least effective against the pod borer. On $14^{\text {th }}$ day after spray revealed that all the treatments proved significantly superior over control.

Carbosulfan (0.52 pod borer larvae plant $\left.^{-1}\right)$ and novaluron ( 0.83 pod borer larvae plant $\left.^{-1}\right)$ were found best treatment in reducing the pod borer population which were statistically at par with each other. The treatments viz. bifenthrin (1.89 pod borer larvae plant $\left.{ }^{-1}\right)$, methyl-o-demeton (2.78 pod borer larvae plant $^{-1}$ ), imidacloprid (3.94 pod borer larvae plant $^{-1}$ ), chlorpyriphos dust (5.05 pod borer larvae plant ${ }^{-1}$ ) and thiamethoxam (6.56 pod borer larvae plant $^{-1}$ ) were statistically at par with each other. Propargite (6.77 pod borer larvae plant ${ }^{-1}$ ) and neem oil (7.74 pod borer larvae plant $^{-1}$ ) were found to be least effective against the pod borer. The pooled data during 2014 and 2015 (Table 3, Second spray) revealed that there was no significant difference between the treatments one day before spray. The observations recorded on $1^{\text {st }}$ day after spray revealed that all the treatments proved significantly superior over control. Carbosulfan (3.44 pod borer larvae plant $^{-1}$ ) was found to be most effective treatment in reducing the pod borer population which was statistically at par with novaluron (3.97 pod borer larvae plant $\left.^{-1}\right)$. The treatments viz. bifenthrin (4.33 pod borer larvae plant $^{-1}$ ), methyl-o-demeton (5.51 pod borer larvae plant $^{-1}$ ), imidacloprid (6.64 pod borer larvae plant $\left.^{-1}\right)$, chlorpyriphos dust (7.71 pod borer larvae plant $^{-1}$ ) and thiamethoxam (8.65 pod borer larvae plant $^{-1}$ ) were statistically at par with each other. Propargite (9.47 pod borer larvae plant ${ }^{-1}$ ) and neem oil (10.66 pod borer larvae plant ${ }^{-1}$ ) were found to be least effective against the pod borer. The observations recorded on $7^{\text {th }}$ day after spray revealed that all the treatments proved significantly superior over control. Carbosulfan (1.60 pod borer larvae plant ${ }^{-1}$ ) was found to be most effective treatment in reducing the pod borer population which was statistically at par with novaluron (2.66 pod borer larvae plant $\left.{ }^{-1}\right)$. The treatments viz. bifenthrin (3.34 pod borer larvae plant $^{-1}$ ), methyl-o-demeton (4.00 pod borer larvae plant ${ }^{-1}$ ), imidacloprid (5.05 pod borer larvae plant $\left.^{-1}\right)$, chlorpyriphos dust (5.43 pod borer larvae plant $^{-1}$ ) and thiamethoxam (6.62 pod borer larvae plant ${ }^{-1}$ ) were statistically at par with each other. Propargite (7.32 pod borer larvae plant ${ }^{-1}$ ) and neem oil (7.91 pod borer larvae plant ${ }^{-1}$ ) were found to be least effective against the pod borer. On $14^{\text {th }}$ day after spray revealed that all the treatments proved significantly superior over control. Carbosulfan (0.29 pod borer larvae plant $\left.^{-1}\right)$ and novaluron $(0.60$ pod borer larvae plant $\left.^{-1}\right)$ were found best treatment in reducing the pod borer population which were statistically at par with each other. 
Table.1 Efficacy of different insecticides against pod borer on marigold (2014)

\begin{tabular}{|c|c|c|c|c|c|c|c|c|c|}
\hline \multirow[t]{3}{*}{ Treatments } & \multirow{3}{*}{$\begin{array}{c}\text { Concentration/ } \\
\text { Dose }\end{array}$} & \multicolumn{8}{|c|}{ Mean Helicoverpa population/plant } \\
\hline & & \multicolumn{4}{|c|}{$1^{\text {st }}$ spray } & \multicolumn{4}{|c|}{$2^{\text {nd }}$ spray } \\
\hline & & 1DBS* & 1DAS* & 7DAS & 14DAS & 1DBS & 1DAS & 7DAS & 14DAS \\
\hline Novaluron 10 EC & 0.100 & $\begin{array}{l}13.36 \\
(3.77)\end{array}$ & $\begin{array}{c}5.56 \\
(2.57)\end{array}$ & $\begin{array}{c}2.61 \\
(1.91)\end{array}$ & $\begin{array}{c}1.05 \\
(1.38)\end{array}$ & $\begin{array}{l}10.00 \\
(3.32)\end{array}$ & $\begin{array}{c}4.67 \\
(2.35)\end{array}$ & $\begin{array}{c}3.65 \\
(2.13)\end{array}$ & $\begin{array}{c}0.61 \\
(1.28)\end{array}$ \\
\hline Carbosulfan $250 \mathrm{EC}$ & 0.003 & $\begin{array}{l}12.67 \\
(3.69)\end{array}$ & $\begin{array}{c}4.65 \\
(2.36)\end{array}$ & $\begin{array}{c}2.00 \\
(1.73)\end{array}$ & $\begin{array}{c}0.67 \\
(1.28)\end{array}$ & $\begin{array}{l}11.00 \\
(3.46)\end{array}$ & $\begin{array}{c}4.32 \\
(2.29)\end{array}$ & $\begin{array}{c}2.21 \\
(1.79)\end{array}$ & $\begin{array}{c}0.26 \\
(1.114)\end{array}$ \\
\hline Imidacloprid $200 \mathrm{SL}$ & 0.008 & $\begin{array}{l}12.61 \\
(3.68)\end{array}$ & $\begin{array}{l}10.08 \\
(3.32)\end{array}$ & $\begin{array}{c}6.67 \\
(2.76)\end{array}$ & $\begin{array}{c}4.33 \\
(2.28)\end{array}$ & $\begin{array}{l}10.67 \\
(3.40)\end{array}$ & $\begin{array}{c}7.29 \\
(2.88)\end{array}$ & $\begin{array}{c}6.00 \\
(2.65)\end{array}$ & $\begin{array}{c}3.68 \\
(1.16)\end{array}$ \\
\hline Bifenthrin $10 \mathrm{EC}$ & 0.002 & $\begin{array}{l}12.00 \\
(3.60)\end{array}$ & $\begin{array}{c}7.33 \\
(2.87)\end{array}$ & $\begin{array}{c}3.65 \\
(2.14)\end{array}$ & $\begin{array}{c}2.00 \\
(1.71)\end{array}$ & $\begin{array}{l}10.34 \\
(3.35)\end{array}$ & $\begin{array}{c}5.00 \\
(2.45)\end{array}$ & $\begin{array}{c}4.38 \\
(2.28)\end{array}$ & $\begin{array}{c}1.33 \\
(1.52)\end{array}$ \\
\hline Methyl-o-Demeton 25 EC & 0.030 & $\begin{array}{l}12.00 \\
(3.60)\end{array}$ & $\begin{array}{c}8.60 \\
(3.10)\end{array}$ & $\begin{array}{c}5.33 \\
(2.49)\end{array}$ & $\begin{array}{c}3.38 \\
(2.02)\end{array}$ & $\begin{array}{l}10.81 \\
(3.40)\end{array}$ & $\begin{array}{c}6.35 \\
(2.71)\end{array}$ & $\begin{array}{c}5.00 \\
(2.45)\end{array}$ & $\begin{array}{c}2.34 \\
(1.82)\end{array}$ \\
\hline Chlorpyriphos dust $\mathbf{1 . 5 \%}$ & $25 \mathrm{~kg} / \mathrm{ha}$ & $\begin{array}{l}13.34 \\
(3.76)\end{array}$ & $\begin{array}{l}10.67 \\
(3.41)\end{array}$ & $\begin{array}{c}8.38 \\
(3.05)\end{array}$ & $\begin{array}{c}5.63 \\
(2.58)\end{array}$ & $\begin{array}{l}10.33 \\
(3.36)\end{array}$ & $\begin{array}{c}8.09 \\
(3.02)\end{array}$ & $\begin{array}{c}6.56 \\
(2.76)\end{array}$ & $\begin{array}{c}5.00 \\
(2.45)\end{array}$ \\
\hline Neem oil & 0.050 & $\begin{array}{l}14.33 \\
(3.91)\end{array}$ & $\begin{array}{l}12.35 \\
(3.65)\end{array}$ & $\begin{array}{r}11.00 \\
(3.46)\end{array}$ & $\begin{array}{c}8.30 \\
(3.05)\end{array}$ & $\begin{array}{l}11.67 \\
(3.56)\end{array}$ & $\begin{array}{l}11.32 \\
(3.51)\end{array}$ & $\begin{array}{c}9.23 \\
(3.16)\end{array}$ & $\begin{array}{c}7.39 \\
(2.87)\end{array}$ \\
\hline Propargite 57 EC & 0.800 & $\begin{array}{l}15.00 \\
(4.00)\end{array}$ & $\begin{array}{l}12.29 \\
(3.65)\end{array}$ & $\begin{array}{l}10.55 \\
(3.31)\end{array}$ & $\begin{array}{c}7.35 \\
(2.85)\end{array}$ & $\begin{array}{l}11.67 \\
(3.56)\end{array}$ & $\begin{array}{l}10.28 \\
(3.37)\end{array}$ & $\begin{array}{c}9.08 \\
(2.26)\end{array}$ & $\begin{array}{c}6.31 \\
(2.71)\end{array}$ \\
\hline Thiamethoxam 25 EC & 0.100 & $\begin{array}{l}12.33 \\
(3.65)\end{array}$ & $\begin{array}{l}11.25 \\
(3.91)\end{array}$ & $\begin{array}{c}9.59 \\
(3.26)\end{array}$ & $\begin{array}{c}7.05 \\
(2.83)\end{array}$ & $\begin{array}{l}10.00 \\
(3.32)\end{array}$ & $\begin{array}{c}9.30 \\
(3.21)\end{array}$ & $\begin{array}{c}7.92 \\
(2.90)\end{array}$ & $\begin{array}{c}5.67 \\
(2.57)\end{array}$ \\
\hline Control & - & $\begin{array}{l}15.68 \\
(4.08)\end{array}$ & $\begin{array}{l}15.01 \\
(4.00)\end{array}$ & $\begin{array}{r}13.33 \\
(3.78)\end{array}$ & $\begin{array}{l}12.69 \\
(3.70)\end{array}$ & $\begin{array}{l}12.33 \\
(3.65)\end{array}$ & $\begin{array}{l}12.05 \\
(3.61)\end{array}$ & $\begin{array}{l}11.00 \\
(3.46)\end{array}$ & $\begin{array}{l}10.33 \\
(3.37)\end{array}$ \\
\hline $\mathrm{CD}(\mathrm{p} \leq \mathbf{0 . 0 5})$ & - & NS & 0.484 & 0.395 & 0.605 & NS & 0.466 & 0.610 & 0.379 \\
\hline SE(m) & & 0.133 & 0.162 & 0.132 & 0.202 & 0.155 & 0.156 & 0.204 & 0.127 \\
\hline
\end{tabular}

*DBS - Days Before Spray, *DAS - Days After Spray; Figures in parenthesis are square $\sqrt{\mathrm{x}}+0.5$ transformed values 
Table.2 Efficacy of different insecticides against pod borer on marigold (2015)

\begin{tabular}{|c|c|c|c|c|c|c|c|c|c|}
\hline \multirow[t]{3}{*}{ Treatments } & \multirow{3}{*}{$\begin{array}{c}\text { Concentration/ } \\
\text { Dose }\end{array}$} & \multicolumn{8}{|c|}{ Mean Helicoverpa population/plant } \\
\hline & & \multicolumn{4}{|c|}{$1^{\text {st }}$ spray } & \multicolumn{4}{|c|}{$2^{\text {nd }}$ spray } \\
\hline & & 1DBS* & 1DAS* & 7DAS & 14DAS & 1DBS & 1DAS & 7DAS & 14DAS \\
\hline Novaluron 10 EC & 0.100 & $\begin{array}{l}10.67 \\
(3.39)\end{array}$ & $\begin{array}{c}4.00 \\
(2.23)\end{array}$ & $\begin{array}{c}2.33 \\
(1.82)\end{array}$ & $\begin{array}{r}0.62 \\
(1.28)\end{array}$ & $\begin{array}{l}11.67 \\
(3.45)\end{array}$ & $\begin{array}{c}3.28 \\
(2.00)\end{array}$ & $\begin{array}{c}1.67 \\
(1.61)\end{array}$ & $\begin{array}{c}0.60 \\
(1.28)\end{array}$ \\
\hline Carbosulfan $250 \mathrm{EC}$ & 0.003 & $\begin{array}{l}12.00 \\
(3.58)\end{array}$ & $\begin{array}{c}3.38 \\
(2.08)\end{array}$ & $\begin{array}{c}1.23 \\
(1.52)\end{array}$ & $\begin{array}{c}0.33 \\
(1.14)\end{array}$ & $\begin{array}{l}10.67 \\
(3.37)\end{array}$ & $\begin{array}{c}2.56 \\
(1.88)\end{array}$ & $\begin{array}{c}1.00 \\
(1.41)\end{array}$ & $\begin{array}{c}0.33 \\
(1.14)\end{array}$ \\
\hline Imidacloprid $200 \mathrm{SL}$ & 0.008 & $\begin{array}{l}10.38 \\
(3.37)\end{array}$ & $\begin{array}{c}7.67 \\
(2.93)\end{array}$ & $\begin{array}{c}5.60 \\
(2.58)\end{array}$ & $\begin{array}{c}3.56 \\
(2.13)\end{array}$ & $\begin{array}{l}11.09 \\
(3.46)\end{array}$ & $\begin{array}{c}6.00 \\
(2.65)\end{array}$ & $\begin{array}{c}4.10 \\
(2.24)\end{array}$ & $\begin{array}{c}2.67 \\
(1.91)\end{array}$ \\
\hline Bifenthrin $10 \mathrm{EC}$ & 0.002 & $\begin{array}{l}10.00 \\
(3.32)\end{array}$ & $\begin{array}{c}5.36 \\
(2.51)\end{array}$ & $\begin{array}{c}3.33 \\
(2.02)\end{array}$ & $\begin{array}{r}1.67 \\
(1.63)\end{array}$ & $\begin{array}{l}11.00 \\
(3.44)\end{array}$ & $\begin{array}{c}3.67 \\
(2.15)\end{array}$ & $\begin{array}{c}2.31 \\
(1.82)\end{array}$ & $\begin{array}{c}1.33 \\
(1.52)\end{array}$ \\
\hline Methyl-o-Demeton 25 EC & 0.030 & $\begin{array}{l}10.00 \\
(3.30)\end{array}$ & $\begin{array}{c}6.68 \\
(2.76)\end{array}$ & $\begin{array}{c}4.50 \\
(2.37)\end{array}$ & $\begin{array}{r}2.28 \\
(1.90)\end{array}$ & $\begin{array}{l}13.33 \\
(3.78)\end{array}$ & $\begin{array}{c}4.67 \\
(2.34)\end{array}$ & $\begin{array}{c}3.00 \\
(2.00)\end{array}$ & $\begin{array}{c}2.00 \\
(1.73)\end{array}$ \\
\hline Chlorpyriphos dust $\mathbf{1 . 5 \%}$ & $25 \mathrm{~kg} / \mathrm{ha}$ & $\begin{array}{l}10.82 \\
(3.39)\end{array}$ & $\begin{array}{c}8.00 \\
(3.00)\end{array}$ & $\begin{array}{c}6.67 \\
(2.74)\end{array}$ & $\begin{array}{r}4.48 \\
(2.36)\end{array}$ & $\begin{array}{l}11.76 \\
(3.56)\end{array}$ & $\begin{array}{c}7.33 \\
(2.85)\end{array}$ & $\begin{array}{c}4.30 \\
(2.29)\end{array}$ & $\begin{array}{c}3.67 \\
(2.15)\end{array}$ \\
\hline Neem oil & 0.050 & $\begin{array}{l}12.00 \\
(3.61)\end{array}$ & $\begin{array}{l}10.67 \\
(3.40)\end{array}$ & $\begin{array}{l}10.00 \\
(3.32)\end{array}$ & $\begin{array}{c}7.24 \\
(2.89)\end{array}$ & $\begin{array}{l}10.81 \\
(3.41)\end{array}$ & $\begin{array}{l}10.00 \\
(3.32)\end{array}$ & $\begin{array}{c}6.74 \\
(2.77)\end{array}$ & $\begin{array}{c}6.33 \\
(2.71)\end{array}$ \\
\hline Propargite 57 EC & 0.800 & $\begin{array}{l}11.78 \\
(3.51)\end{array}$ & $\begin{array}{c}9.64 \\
(3.26)\end{array}$ & $\begin{array}{c}8.38 \\
(3.11)\end{array}$ & $\begin{array}{r}6.20 \\
(2.71)\end{array}$ & $\begin{array}{l}10.00 \\
(3.31)\end{array}$ & $\begin{array}{c}8.67 \\
(3.11)\end{array}$ & $\begin{array}{c}5.42 \\
(2.47)\end{array}$ & $\begin{array}{c}5.00 \\
(2.45)\end{array}$ \\
\hline Thiamethoxam 25 EC & 0.100 & $\begin{array}{l}10.33 \\
(3.37)\end{array}$ & $\begin{array}{r}9.00 \\
(3.16)\end{array}$ & $\begin{array}{c}7.33 \\
(2.88)\end{array}$ & $\begin{array}{r}6.07 \\
(2.65)\end{array}$ & $\begin{array}{c}9.00 \\
(3.16)\end{array}$ & $\begin{array}{c}8.00 \\
(3.00)\end{array}$ & $\begin{array}{c}5.33 \\
(2.49)\end{array}$ & $\begin{array}{c}4.46 \\
(2.34)\end{array}$ \\
\hline Control & - & $\begin{array}{l}12.33 \\
(3.65)\end{array}$ & $\begin{array}{l}11.33 \\
(3.51)\end{array}$ & $\begin{array}{r}10.76 \\
(3.41)\end{array}$ & $\begin{array}{c}9.40 \\
(3.25)\end{array}$ & $\begin{array}{l}11.00 \\
(3.45)\end{array}$ & $\begin{array}{l}10.33 \\
(3.37)\end{array}$ & $\begin{array}{c}9.18 \\
(3.21)\end{array}$ & $\begin{array}{c}9.00 \\
(3.16)\end{array}$ \\
\hline $\mathrm{CD}(\mathrm{p} \leq \mathbf{0 . 0 5})$ & - & NS & 0.380 & 0.527 & 0.489 & NS & 0.503 & 0.723 & 0.411 \\
\hline SE(m) & & 0.170 & 0.127 & 0.176 & 0.163 & 0.238 & 0.168 & 0.242 & 0.137 \\
\hline
\end{tabular}

*DBS - Days Before Spray, *DAS - Days After Spray; Figures in parenthesis are square $\sqrt{\mathrm{x}}+0.5$ transformed values 
Table.3 Efficacy of different insecticides against pod borer on marigold (pooled)

\begin{tabular}{|c|c|c|c|c|c|c|c|c|c|}
\hline \multirow[t]{3}{*}{ Treatments } & \multirow{3}{*}{$\begin{array}{c}\text { Concentration/ } \\
\text { Dose }\end{array}$} & \multicolumn{8}{|c|}{ Mean Helicoverpa population/plant } \\
\hline & & \multicolumn{4}{|c|}{$1^{\text {st }}$ spray } & \multicolumn{4}{|c|}{$2^{\text {nd }}$ spray } \\
\hline & & 1DBS* & 1DAS* & 7DAS & 14DAS & 1DBS & 1DAS & 7DAS & 14DAS \\
\hline Novaluron 10 EC & 0.100 & $\begin{array}{l}12.01 \\
(3.58)\end{array}$ & $\begin{array}{c}5.46 \\
(2.54)\end{array}$ & $\begin{array}{c}2.47 \\
(1.86)\end{array}$ & $\begin{array}{c}0.83 \\
(1.33)\end{array}$ & $\begin{array}{l}10.83 \\
(3.38)\end{array}$ & $\begin{array}{c}3.97 \\
(2.17)\end{array}$ & $\begin{array}{c}2.66 \\
(1.87)\end{array}$ & $\begin{array}{c}0.60 \\
(1.28)\end{array}$ \\
\hline Carbosulfan $250 \mathrm{EC}$ & 0.003 & $\begin{array}{l}12.33 \\
(3.63)\end{array}$ & $\begin{array}{c}4.01 \\
(2.22)\end{array}$ & $\begin{array}{c}1.61 \\
(1.62)\end{array}$ & $\begin{array}{c}0.52 \\
(1.21)\end{array}$ & $\begin{array}{l}10.83 \\
(3.41)\end{array}$ & $\begin{array}{c}3.44 \\
(2.08)\end{array}$ & $\begin{array}{c}1.60 \\
(1.61)\end{array}$ & $\begin{array}{c}0.29 \\
(1.12)\end{array}$ \\
\hline Imidacloprid $200 \mathrm{SL}$ & 0.008 & $\begin{array}{l}11.49 \\
(3.52)\end{array}$ & $\begin{array}{c}8.38 \\
(3.04)\end{array}$ & $\begin{array}{c}6.13 \\
(2.67)\end{array}$ & $\begin{array}{c}3.94 \\
(2.20)\end{array}$ & $\begin{array}{l}10.88 \\
(3.43)\end{array}$ & $\begin{array}{c}6.64 \\
(2.76)\end{array}$ & $\begin{array}{c}5.05 \\
(2.44)\end{array}$ & $\begin{array}{c}3.17 \\
(1.17)\end{array}$ \\
\hline Bifenthrin $10 \mathrm{EC}$ & 0.002 & $\begin{array}{l}11.33 \\
(3.46)\end{array}$ & $\begin{array}{c}5.66 \\
(2.55)\end{array}$ & $\begin{array}{c}3.49 \\
(2.08)\end{array}$ & $\begin{array}{c}1.89 \\
(1.67)\end{array}$ & $\begin{array}{l}10.67 \\
(3.39)\end{array}$ & $\begin{array}{c}4.33 \\
(2.30)\end{array}$ & $\begin{array}{c}3.34 \\
(2.05)\end{array}$ & $\begin{array}{c}1.33 \\
(1.52)\end{array}$ \\
\hline Methyl-o-Demeton 25 EC & 0.030 & $\begin{array}{l}11.00 \\
(3.45)\end{array}$ & $\begin{array}{c}8.13 \\
(3.01)\end{array}$ & $\begin{array}{c}4.91 \\
(2.43)\end{array}$ & $\begin{array}{c}2.78 \\
(1.96)\end{array}$ & $\begin{array}{l}12.07 \\
(3.59)\end{array}$ & $\begin{array}{c}5.51 \\
(2.52)\end{array}$ & $\begin{array}{c}4.00 \\
(2.22)\end{array}$ & $\begin{array}{c}2.17 \\
(1.77)\end{array}$ \\
\hline Chlorpyriphos dust $\mathbf{1 . 5 \%}$ & $25 \mathrm{~kg} / \mathrm{ha}$ & $\begin{array}{l}11.58 \\
(3.52)\end{array}$ & $\begin{array}{l}10.15 \\
(3.33)\end{array}$ & $\begin{array}{c}7.52 \\
(2.89)\end{array}$ & $\begin{array}{c}5.05 \\
(2.47)\end{array}$ & $\begin{array}{l}11.04 \\
(3.46)\end{array}$ & $\begin{array}{c}7.71 \\
(2.93)\end{array}$ & $\begin{array}{c}5.43 \\
(2.52)\end{array}$ & $\begin{array}{l}4.33 \\
(2.3)\end{array}$ \\
\hline Neem oil & 0.050 & $\begin{array}{l}13.16 \\
(3.76)\end{array}$ & $\begin{array}{l}11.62 \\
(3.53)\end{array}$ & $\begin{array}{l}10.50 \\
(3.39)\end{array}$ & $\begin{array}{c}7.74 \\
(2.97)\end{array}$ & $\begin{array}{l}11.24 \\
(3.48)\end{array}$ & $\begin{array}{l}10.66 \\
(3.41)\end{array}$ & $\begin{array}{c}7.91 \\
(2.51)\end{array}$ & $\begin{array}{c}6.32 \\
(2.79)\end{array}$ \\
\hline Propargite 57 EC & 0.800 & $\begin{array}{l}13.39 \\
(3.75)\end{array}$ & $\begin{array}{l}11.48 \\
(3.52)\end{array}$ & $\begin{array}{c}9.46 \\
(3.21)\end{array}$ & $\begin{array}{c}6.77 \\
(2.78)\end{array}$ & $\begin{array}{l}10.83 \\
(3.43)\end{array}$ & $\begin{array}{c}9.47 \\
(3.24)\end{array}$ & $\begin{array}{c}7.32 \\
(2.81)\end{array}$ & $\begin{array}{c}5.65 \\
(2.58)\end{array}$ \\
\hline Thiamethoxam 25 EC & 0.100 & $\begin{array}{l}11.33 \\
(3.51)\end{array}$ & $\begin{array}{l}10.67 \\
(3.38)\end{array}$ & $\begin{array}{c}8.46 \\
(3.07)\end{array}$ & $\begin{array}{c}6.56 \\
(2.74)\end{array}$ & $\begin{array}{c}9.58 \\
(3.24)\end{array}$ & $\begin{array}{c}8.65 \\
(3.10)\end{array}$ & $\begin{array}{c}6.62 \\
(2.69)\end{array}$ & $\begin{array}{c}5.06 \\
(2.45)\end{array}$ \\
\hline Control & - & $\begin{array}{l}14.00 \\
(3.86)\end{array}$ & $\begin{array}{l}13.17 \\
(3.75)\end{array}$ & $\begin{array}{r}12.04 \\
(3.59)\end{array}$ & $\begin{array}{r}11.04 \\
(3.47)\end{array}$ & $\begin{array}{l}11.66 \\
(3.55)\end{array}$ & $\begin{array}{l}11.19 \\
(3.49)\end{array}$ & $\begin{array}{l}10.09 \\
(3.33)\end{array}$ & $\begin{array}{c}9.66 \\
(3.26)\end{array}$ \\
\hline $\mathrm{CD}(\mathrm{p} \leq \mathbf{0 . 0 5})$ & - & NS & 0.432 & 0.461 & 0.547 & NS & 0.484 & 0.666 & 0.395 \\
\hline SE(m) & & 0.151 & 0.144 & 0.154 & 0.182 & 0.196 & 0.162 & 0.223 & 0.132 \\
\hline
\end{tabular}

*DBS - Days Before Spray, *DAS - Days After Spray; Figures in parenthesis are square $\sqrt{\mathrm{x}}+0.5$ transformed values 
The treatments viz. bifenthrin (1.33 pod borer larvae plant $\left.{ }^{-1}\right)$, methyl-o-demeton (2.17 pod borer larvae plant $^{-1}$ ), imidacloprid (3.17 pod borer larvae plant $^{-1}$ ), chlorpyriphos dust (4.33 pod borer larvae plant $^{-1}$ ) and thiamethoxam (5.06 pod borer larvae plant ${ }^{-1}$ ) were statistically at par with each other. Propargite (5.65 pod borer larvae plant ${ }^{-1}$ ) and neem oil (6.32 pod borer larvae plant ${ }^{-1}$ ) were found to be least effective against the pod borer. The present results are in agreement with Yogeeswarudu and Krishna (2014) who reported that indoxacarb 14.5 SC @ $0.5 \mathrm{ml} / 1$ was found best with minimum population of $H$. armigera among different treatments viz., indoxacarb 14.5 SC @ $0.5 \mathrm{ml} / 1$, profenofos 50 EC @ 2.0 ml/1, imidacloprid 17.8 SL @ 1 m/l, novaluron 10 EC @ 1.5 ml/1, fipronil 5 SC @ $2.0 \mathrm{ml} / 1$ and lambda cyhalothrin 5 EC @ 1 $\mathrm{ml} / \mathrm{l}$. The results of present studies are in agreement with Gandhi et al., (2013) who evaluated the bio-efficacy of spinosad 45SC $(0.1 \mathrm{ml} / \mathrm{L})$, cypermethrin 10EC $(0.5 \mathrm{ml} / \mathrm{L})$, novaluran 10EC $(1 \mathrm{ml} / \mathrm{L})$, azardirachtin $5 \%$ and Bacillus thringiensis $(1 \mathrm{ml} / \mathrm{L})$ insecticides against $H$. armigera and reported that spinosad 45SC $(0.1 \mathrm{ml} / \mathrm{l})$, novaluran 10EC(1ml/1) and Azardirachtin $5 \%$ emerged as superior in reducing the population of the insect. Our results are in contradiction with Hussain and Bilal (2007) who reported that among the treatments imidacloprid at $0.03 \%$ proved more effective followed by deltamethrin and fluvalinate.

\section{References}

Anonymous, 2014. Pests of ornamental plants. www. tnau. ac. In / eagri / eagri 50 / ENTO 331 / lecture31/lec031.pdf. Accessed on 28/1/14.

Anonymous, 2016. Floriculture and Seeds. APEDA, Government of India. http://www.apeda.gov.in / apedawebsite /six_head_product / floriculture.htm

Bhattacharjee, S.K., 2001. "Periurban Floriculture and Quality of Life", Indian Horti., 45(4):33-35.

Gandhi, B.K., Shekharappa, K. and Balikai, R.A. 2013. Bio-efficacy of insecticides in management of Helicoverpa armigera (Hübner) in Kharif Sorghum. Annl. Plant Prot. Sci., 21(1):83-86.

Hussain, B., and Bilal, S. 2007. Efficacy of different insecticides on tomato fruit borer Helicoverpa armigera. J. Entomol., 4(1):64-67.

Pandey, R.K., Dogra, S., Sharma, J.P., Jambal, S. and Bhat, D.J. 2010. Performance of Gladiolus cultivars under Jammu conditions. J. Res. SKUAST., 2:210-214.

Yogeeswarudu, B., and Krishna, V.K. 2014. Field studies on efficacy of novel insecticides against Helicoverpa armigera (Hubner) infesting on Chickpea. J. Entomol. Zool. Stud., 2(4):286-289.

\section{How to cite this article:}

Suheel Ahmad Ganai, Hafeez Ahmad, Sonika Sharma, Nadeya Khaliq, Thanlass Norboo, Divya Chaand and Shallu Raina. 2017. Management of Pod Borer (Helicoverpa armigera Hubner) Infesting Marigold (Tagetes erecta). Int.J.Curr.Microbiol.App.Sci. 6(9): 142-148. doi: https://doi.org/10.20546/ijcmas.2017.609.017 